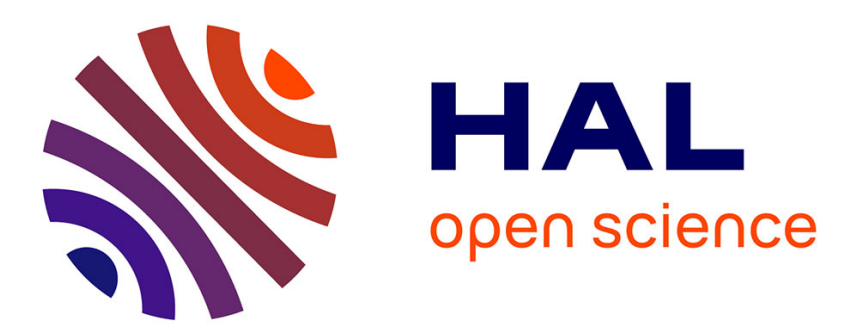

\title{
Étude théorique et expérimentale des transitions à plusieurs quanta entre les sous-niveaux Zeeman d'un atome
}

\author{
J.M. Winter
}

\section{To cite this version:}

J.M. Winter. Étude théorique et expérimentale des transitions à plusieurs quanta entre les sousniveaux Zeeman d'un atome. J. Phys. Radium, 1958, 19 (11), pp.802-805. 10.1051/jphysrad:019580019011080200 . jpa-00235935

\section{HAL Id: jpa-00235935 https://hal.science/jpa-00235935}

Submitted on 1 Jan 1958

HAL is a multi-disciplinary open access archive for the deposit and dissemination of scientific research documents, whether they are published or not. The documents may come from teaching and research institutions in France or abroad, or from public or private research centers.
L'archive ouverte pluridisciplinaire HAL, est destinée au dépôt et à la diffusion de documents scientifiques de niveau recherche, publiés ou non, émanant des établissements d'enseignement et de recherche français ou étrangers, des laboratoires publics ou privés. 


\title{
ÉTUDE THÉORIQUE ET EXPÉRIMENTALE DES TRANSITIONS A PLUSIEURS QUANTA ENTRE LES SOUS-NIVEAUX ZEEMAN D'UN ATOME
}

\author{
Par J. M. WINTER, \\ Laboratoire de Physique, E. N. S., Paris et C. E. A., Saclay.
}

Résumé. - Des expériences récentes ont montré que l'on peut observer des transitions dipolaires magnétiques n'obéissant pas aux règles de sélection $\Delta m= \pm 1,0$ ( $m$ étant la projection du moment angulaire total de l'atome sur le champ statique $H_{0}$ ).

L'influence d'un champ magnétique oscillant de pulsation $\omega$ sur les sous-niveaux Zeeman d'un atome a été étudiée d'une façon assez détaillée. Trois cas sont à distinguer :

1) Le champ perturbateur est un champ tournant perpendiculaire à $H_{0}$ (pulsation $\omega$, amplitude $H_{1}$ ).

Il y a résonance chaque fois que la pulsation $\omega$ obéit à l'équation

$$
E(m)-E\left(m^{\prime}\right)=\hbar \omega\left(m-m^{\prime}\right)
$$

$E(m)$ étant l'énergie d'un état $m$. sition.

2) Le champ oscillant est un champ elliptique (d'orientation quelconque par rapport à $H_{0}$ ).

Un nouveau type de résonance apparaît alors. Supposons par exemple $J=1 / 2(I=0)$; en plus de la résonance $\hbar \omega=E(+1 / 2)-E(-1 / 2)$ obtenu dans le $1^{\mathrm{er}}$ cas, on montre qu'il y a résonance chaque fois que la relation $p \hbar \omega=E(+1 / 2)-E(-1 / 2)$ est vérifiée $(p$ entier quelconque).

L'existence de telles transitions peut se comprendre en invoquant la loi de conversation. du moment angulaire total du système constitué par l'atome et les photons de radiofréquence.

3) Il y a dēux champs oscillants à des fréquences différentes $\omega$ et $\omega^{\prime}$ : Dans le cas $J=1 / 2$ $I=0$. Il y a résonance chaque fois que

( $p, q$ entier positif ou négatif).

$$
E(1 / 2)-E(-1 / 2)=\hbar\left(p \omega+q \omega^{\prime}\right)
$$

- Dans les trois cas, nous avons calculé les déplacements, largeurs et intensités des résonances en fonction de l'inténsité $H_{1}$ du champ de radiofréquence.

Ces théories ont été vérifiées expérimentalement sur l'état fondamental de l'atome de sodium $(J=1 / 2, I=3 / 2)$. Nous avons employé une vapeur saturante de sodium orientée optiquement en présence d'un gaz étranger. Les résonances sont détectées optiquement. Les vérifications ont porté sur les points suivants :

1) Mise en évidence des résonances multiples, 2) position des résonances, 3) variation de leur largeur et de leur intensité avec $H_{1}$.

En particulier nous avons vérifié que les déplacements des résonances varient toujours comme $H_{1}^{2}$ alors que la largeur d'une résonance utilisant $p$ quanta varie comme $H_{1}^{p}$. L'accord entre la théorie et l'expérience est bon. Nous avons également mesuré les largeurs de raies pour de faibles niveaux de radiofréquence ce qui nous permet de déterminer la largeur de raie provenant de la relaxation. Elle est de l'ordre de 200 cycles.

Abstract. - Recent experiments have shown that magnetic dipole transitions are observed, in which the selection rule $\Delta m= \pm 1,0$, is not followed.

Transitions induced by a magnetic oscillating field $H_{1}$ have been studied in the following hypotheses.

a.) $H_{1}$ is a rotating field, perpendicular to $H_{0}$, a transition is obtained when ever

$m-m^{\prime}=0, \pm 1, \pm 2$, etc..

$$
E_{m}-E_{m^{\prime}}=\hbar \omega\left(m-m^{\prime}\right)
$$

b) $H_{1}$ is elliptical (the plane of the ellipse being arbitrary). In the case of a 2 level system $(J=1 / 2)$, resonances are observed whenever

$p$, any integer.

$$
E_{1 / 2}-E_{-1 / 2}=p . \hbar \omega
$$

There is, of course, conservation of energy and of angular momentum in these transitions.

c) 2 oscillating fields are present $\omega$ and $\omega^{\prime}$. In the case $J=1 / 2$, there is resonance when

$$
E_{1 / 2}-E_{-1 / 2}=\hbar\left(p \omega+q \omega^{\prime}\right)
$$

$p, q$, positive or negative integers.

In each case, we have computed energy displacements, line widths and line intensities as a function of $H_{1}$

All the above predictions have been observed experimentally, on $\mathrm{Na}$ atoms. We used optical pumping and optical selection. The buffer gas was argon at $0,3 \mathrm{~mm}$.

We verified that line displacements were a linear function of $H_{1}^{2}$, whereas line widths are linear functions of $H_{1}^{p}$ for a $p$ quanta line.

The ultimate width of our lines at low $r$-f levels was of the order of 200 cycles. 
Étude théorique. - Les résonances magnétiques entre les sous-niveaux de l'état fondamental d'un atome sont des transitions magnétiques dipolaires. Elles obéissent aux règles de sélection

$$
\Delta J= \pm 1,0 \quad \Delta m= \pm 1,0
$$

( $J$ étant le moment angulaire total et $m$ sa projection sur le champ magnétique statique $H_{0}$ ).

De nombreuses expériences ont montré que l'on peut observer des résonances n'obéissant pas à la règle de sélection $\Delta m= \pm 1 ; 0[1]$. Nous avons donc repris l'étude théorique de l'interaction entre un champ oscillant et les sous-niveaux de l'atome. Trois cas sont à distinguer :

10 LE CHAMP OSCILLANT EST UN CHAMP TOURNANT PERPENDiculaire a $H_{0}$ (PUlsation $\omega$, AMPLITUDE $H_{1}$ ).

Supposons, pour simplifier, que l'atome possède 3 niveaux non équidistants ( $m$ étant un bon nombre quantique). Le calcul montre qu'en plus des deux résonances $m=1 \longleftrightarrow m=0$ et $m=0 \leftarrow \rightarrow m=-1$, il apparaît une résonance $\Delta m=2$ quand $\omega$ satisfait la relation :

$2 \hbar \omega=E(1)-E(-1) \quad(E(m)$ énergie de l'état $m)$.

La largeur de cette résonance est proportionnelle à $H_{1}^{2}$ /e (e étant l'écart en énergie entre le

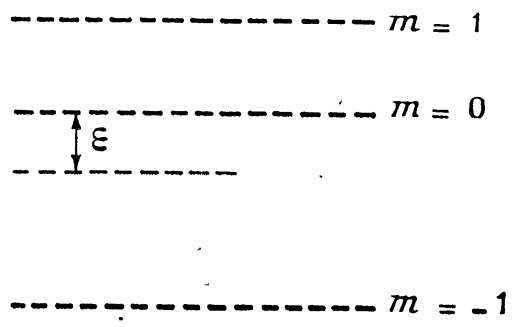

Fig. 1.

niveau $m=0$ et un niveau équidistant des niveaux $m=1$ et $m=-1$ ) [2], [3]. La largeur des résonances $\Delta m=1$ est proportionnelle à $H_{1}$. On peut dire que dans la transition $\Delta m=2$, le système atomique absorbe deux photons de radiofréquence, chaque photon amène une énergie $\hbar \omega$, et augmente $m$ d'une unité. Plus généralement, des résonances apparaissent chaque fois que $\omega$ obeiit à l'équation

$$
E\left(m^{\prime}\right)-E(m)=\left(m^{\prime}-m\right) \hbar \omega .
$$

Les probabilités de transitions se calculent au voisinage de chaque résonance (on suppose que les distances entre résonances sont grandes devant leur largeur).

$$
\begin{gathered}
P\left(m, m^{\prime}, t\right)=\frac{4 K_{m m^{\prime}}^{2}}{a^{2}} \sin ^{2} \frac{a t}{2} \\
a^{2}=\left[\frac{E_{m^{\prime}}-E_{m}}{\hbar}+\delta_{m m^{\prime}}-\left(m-m^{\prime}\right) \omega\right]^{2}+4 K_{n t m^{\prime}}^{2}
\end{gathered}
$$

$K_{m m^{\prime}}$ est une quantité proportionnelle à $H_{1}^{i m-m^{\prime} \mid}$, elle donne l'élargissement produit par la radiofréquence ; $\delta_{m m^{\prime}}$ est un terme proportionnel à $H_{1}^{2}$ qui conduit à un léger déplacement de la fréquence de résonance. Dans une transition $\Delta m=p$, le système absorbe $p$ photons.

$2^{\circ}$ LE CHAMP OSCILLANT EST UN CHAMP ELLIPTIQUE (D'ORIENTATION QUELCONQUE PAR RAPPORT A $H_{0}$ ).

Étudions, pour simplifier, un atome avec $J=1 / 2$ (sans spin nucléaire). Si le champ est tournant, on observe une résonance

$$
\hbar \omega=E\left(\frac{1}{2}\right)-E\left(-\frac{1}{2}\right)
$$

Si le champ est quelconque, il y a résonance quand

$p \hbar \omega=E(1 / 2)-E(-1 / 2) \quad(p$ entier quelconque).

On peut donner deux explications simples de cette relation.

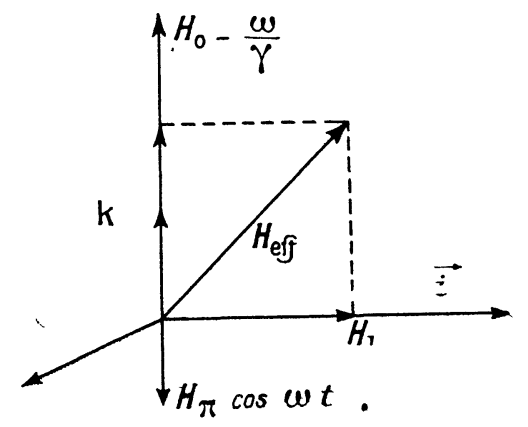

FIg. 2.

a) Un champ oscillant quelconque se décompose en deux champs tournants, l'un droit $\left(\sigma^{+}\right)$, l'autre gauche $\left(\sigma^{-}\right)$perpendiculaires à $H_{0}$ et en un champ oscillant parallèle à $H_{0}(\pi)$. Il y a donc 3 sortes de photons de radiofréquence. Les photons $\sigma^{+}$ ajoutent une unité.à $m$, les $\sigma^{-}$retranchent une unité, les $\pi$ ne changent pas $m$. Il est alors possible de satisfaire le principe de conservation du moment angulaire total en échangeant un nombre quelconque de photons entre l'atome et le champ de radiofréquence.

b) L'évolution du système atomique peut se déduire de l'évolution d'un moment magnétique $\stackrel{\vec{M}}{\longrightarrow}$ obéissant à l'équation

$$
\frac{\mathrm{d} \vec{M}}{\mathrm{~d} t}=\gamma \vec{M} \wedge \vec{H}
$$

Supposons, par exemple, que le champ oscillant se décompose en un champ circulaire droit $H_{1}$ et en un champ $H_{\pi} \cos \omega t$, parallèle à $H_{0}$. Si l'on passe dans le référentie] tournant lié à $H_{1}$ le champ $H_{0}$ devient $H_{0}-\omega / \%, H_{1}$ devenant cons- 
tant. Ces deux champs s'ajoutent pour donner un champ $H_{\text {eff }}$

$$
H_{\mathrm{eff}}=\frac{1}{\gamma}\left\{\left(\omega_{0}-\omega\right) \vec{k}+\gamma H_{1} \overrightarrow{i^{\prime}}\right\} \quad \omega_{0}=\gamma H_{0} .
$$

Le champ $H \pi$ possède des composantes tournantes perpendiculaires à $H_{\text {eff }}$ (vitesse angulaire $\omega$ ).

Il y aura résonance quand ذ: $\omega \simeq \gamma H_{\text {eff }}$ en particulier quand $2 \omega \simeq \omega_{0}$.

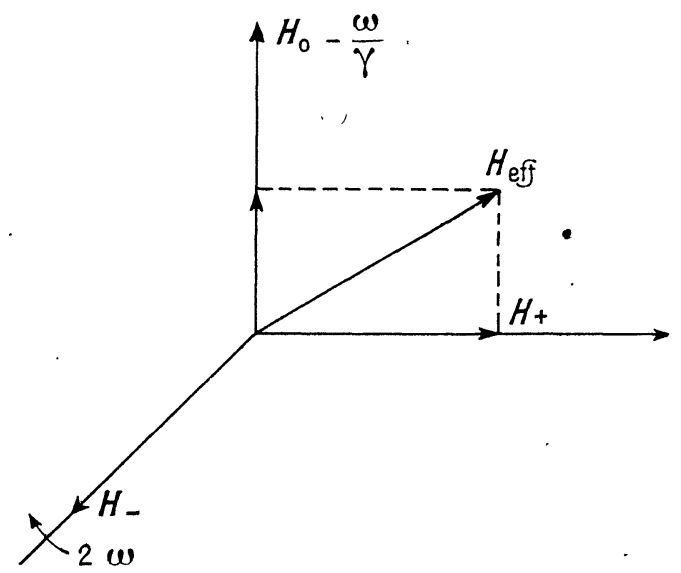

Fiti. 3.

Un peut continuer la discussion et démontrer l'existence de résonances pour $p \omega=\omega_{0}$.

Supposons maintenant que $H \pi=0$, mais ajoutons un champ tournant gauche. Quand on passe dans le référentiel tournant lié au champ circulaire droit $H_{+}$, le champ tournant gauche $H_{-}$tourne à la vitesse angulaire $2 \omega$ ( $f$ ig. 3).

Composons $H_{+}$et $H_{0}-\omega / \gamma$ pour obtenir $H_{\text {eff }}$. Le champ gauche $H_{-}$possède alors une composante circulaire droite perpendiculaire à $H_{\text {eff }}$ et il $\mathrm{y}$ a résonance quand

$$
2 \omega \sim \omega_{0}+\omega .
$$

Plus généralement, il y a résonance quand

$$
\omega_{0} \sim(2 p+1) \omega .
$$

Cette propriété s'explique aisément en employant le langage des photons. Il n'y a plus de photons $\pi(\Delta m=0)$. On ne peut induire une transition $\Delta m=1$ qu'avec un nombre impair de photons $\sigma^{+}$ et $\sigma$, par exemple $p+1$ photons $\sigma^{+}$et $p$ photons $\sigma$.

On peut calculer les probabilités de transitions au voisinage de ces résonances. Elles subissent des déplacements proportionnels à $H_{1}^{2} / \omega$ analogues au déplacement Bloch-Siegert [6], [7].

Une résonance à $p$ quanta possède une largeur proportionnelle à $H_{1}^{p} / \omega^{p-1}$.

Ces résultats se généralisent si $J>1 / 2$ et s'jil y a deux moments angulaires $I$ et $J$.
30 IL Y A DEUX CHAMPS DE FRÉQUENGES DIFFÉRENTES $\left(\omega\right.$ et $\left.\omega^{\prime}\right)$. (ON Suppose $J=1 / 2, I=0$.)

La condition de résonance est alors

$$
E(1 / 2)-E(-1 / 2)=h\left(p \omega+q \omega^{\prime}\right)
$$

$p$ et $q$ entier positif ou négatif.

Les calculs de déplacements et de largeurs de raies sont semblables à ceux du cas précédent. La majeure partie des vérifications expérimentales a 'été effectuée sur l'état fondamental de l'atome de sodium ${ }^{2} S_{1 / 2}, J=1 / 2, I=3 / 2$.
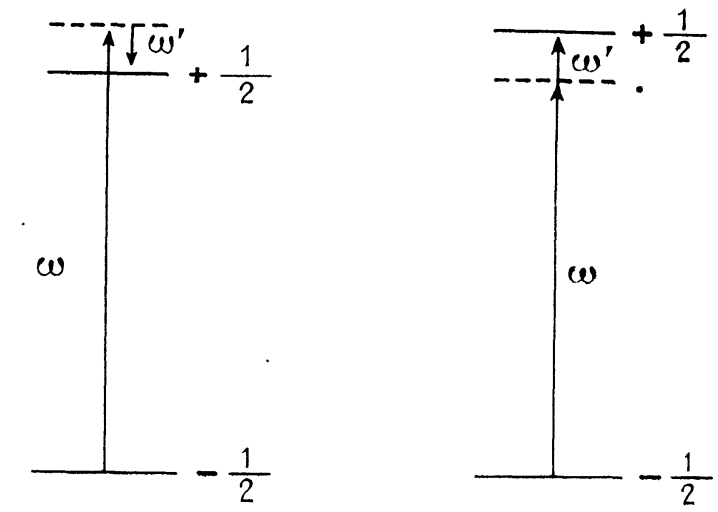

FIri. I.

Résultats expérimentaux. - $-u$ ) Nous avons l'abord mis en évidence les divers types de résonances multiples. .b) Nous avons ensuite étudié leurs déplacements en fonction de l'amplitude du champ de radiofréquence, vérifié que ces dépla-

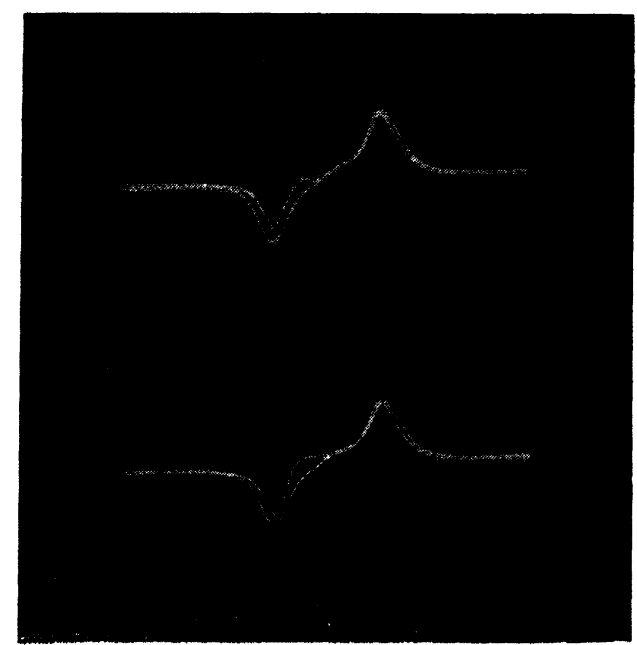

Fig. 5. - En abscisse $H_{0}$, en ordonnée le niveau de $\omega$.

cements sont proportionnels à $H_{1}^{2}$ (les rapports des déplacements des différentes raies sont en a ccord avec les rapports théoriques). $c$ ) Nous avons vérifié que la largeur d'une résonance utilisant $p$ quanta varie comme $H_{1}^{p}$. 
Nous avons observé que certaines résonances disparaissent quand le champ oscillant est rigoureusement perpendiculaire au champ statique. L'accord entre la théorie et l'expérience est bon.

Résultats nouveaux. - Nous avons observé récemment des résonances multiples entre les deux niveaux de résonance électronique du diphénylpycrilhydrazyl. Nous disposons de deux fréquences $\omega \sim 9000 \mathrm{MHz}, \omega^{\prime} \sim 20 \mathrm{MHz}$.

On observe les résonances

$$
\omega \pm \omega^{\prime}=\gamma H_{0} .
$$

La fréquence $\omega^{\prime}$ est produite par un oscillateur du type autodyne, le niveau d'oscillation varie quand il y a absorption ou émission de photons à la fréquence $\omega^{\prime}$. Quand $\omega+\omega^{\prime}=\gamma H_{0}$, il y a absorption d'énergie à la fréquence $\omega^{\prime}$; quand $\omega-\omega^{\prime}=\gamma H_{0}$, il y a émission (fig. 4 et 5).

Ainsi, il est possible de détecter par un procédé radioélectrique une résonance paramagnétique dans un champ élevé sans dispositif de mesure en hyperfréquence.

\section{BIBLIOGRAPHIE}

[1] Brossel (J.), Gagnac (B.), Kastler (A.), J. Physique Rad., 1954, 15, 6. Kusch (P.), Phys. Rev., 1954, 93, 1022. Anderson (W.), Phys. Rev., 1956, 104, 850.

[2] Besset (G.), Horowitz, Messiah, Winter, J. Physique Rad., 1954, 15, 251.

3] Salwen (H.), Phys. Rev., 1955, 99, 1274.

[4] Winter (J.), C. R. Acad. Sc., 1955, 241, 600.

55 Rabi (I.), Ramsey (N.), Schwinger (J.), Rev. Mod. Physics, 1954, 26, 168.

[6] Bloch (F.), Siegert (A.), Phys. Rev., 1940, 57, 522.

[7] Winter (J.), C. R. Acad. Sc., 1955, 241, 375-377. 